\title{
$\mathrm{M}|\mathrm{R}| \mathrm{S}$ Internet Journal Nitride Semiconductor Research
}

\section{Effect of Photo-Assisted RIE Damage on GaN}

\author{
Z. Mouffak ${ }^{12}$, N. Medelci-Djezzar ${ }^{1}$, C. Boney ${ }^{1}$, A. Bensaoula ${ }^{1}$ and L. Trombetta ${ }^{2}$ \\ ${ }^{1}$ Texas Center of Superconductivity and Advanced Materials, Department of Physics, University of Houston, \\ ${ }^{2}$ Department of Electrical and Computer Engineering, University of Houston,
}

(Received Friday, July 11, 2003; accepted Friday, September 26, 2003)

Reactive Ion Etching (RIE) and Photo-Assisted RIE (PA-RIE) induced damage in GaN using simple Schottky structures and a $\mathrm{BCl}_{3} / \mathrm{Cl}_{2} / \mathrm{N}_{2}$ gas mixture have been investigated. Schottky diode I-V characteristics following different RF powers and exposure times show significant changes caused by damage. This damage results in a reduction of the reverse breakdown voltage $V_{B}$ in $n$-type GaN and an increase in $\mathrm{V}_{\mathrm{B}}$ for p-type $\mathrm{GaN}$. Our preliminary data on the PA-RIE process points to much reduced damage levels compared to conventional RIE. This result may be due to a change in surface chemistry or to a photo-enhanced diffusion of defects into the GaN layer, leaving a cleaner nearsurface region.

\section{Introduction}

There is currently much interest in the development of electronic and opto-electronic devices based on IIINitride semiconductor alloys (principally the binary and ternary alloys of $\mathrm{Ga}$, In, and $\mathrm{Al}$ with $\mathrm{N}$ ). These materials have direct band gaps ranging from $0.7 \mathrm{eV}$ for $\mathrm{InN}$ to $6.2 \mathrm{eV}$ for AlN, and high thermal conductivity. Thus they hold promise for a variety of applications that are difficult or impossible to achieve with other materials, including transistors operating at high temperature or high power, and light emitters/detectors in the blue/UV spectrum. [1] [2] [3]

In fabricating devices from the III-Nitrides, the use of plasma etching is critical for obtaining anisotropic etch profiles and sufficiently high etch rates. However, plasma techniques induce lattice damage in the semiconductor, resulting in electrically active defects in the near-surface region of the device. Such defects degrade device performance by causing excessive leakage current and reduced optical efficiency, and therefore much effort is expanded to better understand and control the etch process.

Studies using inductively coupled plasmas (ICP) show high damage levels that are partially removed by wet etching [4]. While this approach might be adequate for devices fabricated using relatively thick top active layers (i.e. LEDs and some lasers), it is not acceptable in others, where removal of a relatively thick layer after plasma etching may not be possible. This issue will become more acute as device geometries shrink and sophisticated heterostructures (e.g., the HEMT) become necessary. Furthermore, wet etching is always a potential source of additional surface contamination.

Development of an all-dry etching technique that minimizes plasma damage would thus be highly desirable. For this purpose, photo-assisted RIE (PA-RIE) processes have been investigated for a variety of materials [5] [6] [7]. PA-RIE requires minor or no modification to the reactor geometry and no incorporation of new hardware into the plasma environment. As a result current plasma etching reactors can be easily modified if such a technology is proven superior. A study of the GaN etched surface composition using XPS [7] showed that using a photo-assisted RIE process reduces the surface nitrogen depletion; which could be an indication of reduced etching damage on the surface. In this paper, we study the RIE induced damage in $\mathrm{GaN}$ using simple Schottky structures and a $\mathrm{BCl}_{3} / \mathrm{Cl}_{2} / \mathrm{N}_{2}$ gas mixture. We also perform a comparative investigation of RIE and PARIE.

\section{Experimental}

The $\mathrm{n}$ and $\mathrm{p}-\mathrm{GaN}$ Schottky structures are shown schematically in Figure 1. The GaN films were grown by RF-plasma source molecular beam epitaxy (RF-MBE) on (0001) sapphire substrates. An AlN buffer layer is grown on the substrate and is followed by an unintentionally doped $\mathrm{GaN}$ layer and finally the active $\mathrm{GaN}$ layer. Silicon and magnesium are used as n-type $\left(\sim 3.4 \times 10^{17} \mathrm{~cm}^{-3}\right)$ and p-type $\left(\sim 10^{17} \mathrm{~cm}^{-3}\right)$ dopants, 
respectively. Etching is performed in an Oxford Plasma Technology $80 \mu \mathrm{p}$ RIE reactor. The base pressure of the reactor is $1 \times 10^{-6}$ Torr. In the PA-RIE process, light from a Xe arc lamp is directed through a UV lens. After reflection by a UV mirror and collimation by a second UV lens, it impinges at normal incidence onto the sample through a quartz window in the RIE chamber. A crown glass window and a water IR filter are set in the light path in order to filter the UV and the IR portion of the spectrum respectively. The light power density is 38 $\mathrm{mW} / \mathrm{cm}^{2}$ at the sample, with a spot diameter of $2 \mathrm{~cm}$ (much larger light sources can be developed for industrial applications). Ti/Au and $\mathrm{Ni} / \mathrm{Au}$ ohmic contacts were deposited by e-beam evaporation on $\mathrm{n}$ and p-type $\mathrm{GaN}$, respectively. The $\mathrm{n}$ and p-type $\mathrm{GaN}$ films were etched in $10 \mathrm{sccm} \mathrm{Cl}_{2} / 10 \mathrm{sccm} \mathrm{BCl} / 10 \mathrm{sccm} \mathrm{N} \mathrm{N}_{2}$ at 30 mTorr and at powers ranging from 50 to $200 \mathrm{~W}$ and times ranging from 30 to 120 s for $n-G a N$ samples, and at $2 \mathrm{~min}$ for all $\mathrm{p}-\mathrm{GaN}$ samples. Finally, $\mathrm{Pt} / \mathrm{Au}$ and $\mathrm{Si} / \mathrm{Ti} /$ Au Schottky contacts were deposited by e-beam evaporation on $\mathrm{n}$ and p-type $\mathrm{GaN}$, respectively.

\section{Results and Discussion}

Figure 2 shows the IV characteristics of Schottky structures made on $\mathrm{n}-\mathrm{GaN}$ and exposed to RIE and PA-RIE for different RF powers. In each RIE run, a sample was put under light for PA-RIE etching, and another sample was put far from it in a dark spot for RIE etching. In both cases there is less damage with higher RF powers. This is possibly due to that for short plasma exposure times, an increase in power increases the removal of the accumulated damage of the first seconds of etch. First there is a large accumulation of damage at the beginning of the etching process. For a low RF power $\sim 50 \mathrm{~W}$ (DC self bias potential of $-100 \mathrm{~V}$ ), ions are energetic enough to create surface damage but the etch rate is very small. In fact, a lower power creates more damage since the plasma is not completely dissociated and the density of the active radicals is too low for substantial reaction with the surface. For a higher power, and for the same short time, ions have enough energy to create damage but are also reactive enough to partially remove the accumulated damage. At higher powers, for the same short etching time, the sputtering mechanism becomes more important than the chemical process, resulting in increased damage.

We also performed RIE and PA-RIE experiments for longer exposure times at fixed RF power. Figure 3 shows the IV characteristics for a sample processed by RIE and PA-RIE at $200 \mathrm{~W}$ RF power and for $2 \mathrm{~min}$. The control sample is shown for reference. We can clearly see that the IV of the PA-RIE exposed structure is less leaky than that of the RIE exposed one, which suggests that we have less damage in a photo-assisted process under these conditions.

The variation of the reverse breakdown voltage $\left(\mathrm{V}_{\mathrm{B}}\right)$ and the turn-on forward voltage $\left(\mathrm{V}_{\mathrm{F}}\right)$ with the RF power for samples etched for 30s is shown in Figure 4. Figure 5 shows the dependence of the same parameters with the time of exposure to the plasma for a fixed RF power of $200 \mathrm{~W} . V_{B}$ and $V_{F}$ have been defined at a current of $0.1 \mathrm{~mA}$ and $+0.1 \mathrm{~mA}$ respectively. We can see from those figures that PA-RIE becomes less damaging than RIE beyond a threshold RF power and exposure time.

In addition to the competition between damage production and removal, surface defects have been observed to diffuse into the bulk in GaN [4]. If optical excitation increases the diffusion coefficient of surface defects, such defects may not be detected by I-V characterization, which is sensitive only to near surface defects. Deep level transient spectroscopy (DLTS) experiments could give more decisive information about defects. These experiments are ongoing.

Our preliminary electrical data (using Schottky diodes) on plasma-induced damage in p-type $\left(10^{17} \mathrm{~cm}^{-}\right.$ $\left.{ }^{3}\right) \mathrm{GaN}$ as a function of RF power show an enhancement of the barrier height for $\mathrm{p}$ type $\left(10^{17} \mathrm{~cm}^{-3}\right) \mathrm{GaN}$ and so, an increase in $V_{B}$ as seen in Figure 6. That behavior is similar to that already demonstrated in ICP treated p$\mathrm{GaN}$, which was attributed to the creation of shallow donors related to a nitrogen vacancy [8]. These samples were etched for 2 minutes and the competition between etching rate and damage removal is again sensed here: From the IV characteristics we see that $50 \mathrm{~W}$ RF power induced more damage than $100 \mathrm{~W}$ but less than $200 \mathrm{~W}$. We also performed the etching of $\mathrm{p}$-GaN under PA-RIE for 200W RF power. Our results suggest that less damage is induced by PA-RIE (using the filtered Xe lamp) than by conventional RIE performed under the same conditions (200 W RF power and corresponding $-300 \mathrm{~V}$ dc self-bias), and for the same etch time ( $2 \mathrm{~min})$. The reverse breakdown voltage defined at $-50 \mu \mathrm{A}$ showed a value of $-7.6 \mathrm{~V}$ for the PA-RIE etched structures versus $12.3 \mathrm{~V}$ for diodes made on samples etched with RIE.

\section{Conclusion}

Dramatic changes occur in reverse breakdown voltage $\mathrm{V}_{\mathrm{B}}$ and forward turn-on voltage $\mathrm{V}_{\mathrm{F}}$ for GaN Schottky diodes after RIE or PA-RIE exposure. Damage accumulates in the near-surface region at short exposure times and low RF power energy, creating an even more degraded surface than at higher RF powers and exposure times. Less damage with PA-RIE is reported for relatively high RF powers and longer exposure times. 


\section{ACKNOWLEDGMENTS}

This work was supported by funds from a NASA ccoperative \#NCC8-127 to TcSAM, an Institute of Space Systems and Operations grant, a Texas Space Grant Consortium grant, and a Texas Advanced Technology Program Grant \#1-1-32061

\section{REFERENCES}

[1] H. Amano, M. Kito, K. Hiramatsu, I. Akasaki, Jpn. J. Appl. Phys. 28, L2112 (1989).

[2] S. Nakamura, M. Senoh, S. Nagahama, N. Iwasa, T. Yamada, T. Matsushita, H. Kiyoku, Y. Sugimoto, T. Kozaki, H. Umemoto, M. Sano, K. Chocho, Jpn. J. Appl. Phys. 36, L1568 (1997).

[3] M. Asif Khan, J. N. Kuznia, A. R. Bhattarai, D. T. Olson, Appl. Phys. Lett. 62, 1786-1787 (1993).

[4] X. A. Cao, S. J. Pearton, G. T. Dang, A. P. Zhang, F. Ren, J. M. Van Hove, IEEE Trans. Electr. Dev. 47, 1320-1324 (2000).

[5] N Medelci, A Tempez, D Starikov, N Badi, I Berishev, A Bensaoula, J. Electron. Mater. 29, 1079-1083 (2000).

[6] N Medelci, A Tempez, I Berishev, D Starikov, A Bensaoula, Mater. Res. Soc. Symp. Proc. 572, 535-540 (1999).

[7] A Tempez, N Medelci, N Badi, I Berishev, D Starikov, A Bensaoula, J. Vac. Sci. Technol. A 17, 2209-221 (1999).

[8] XA Cao, SJ Pearton, AP Zhang, GT Dang, F Ren, RJ Shul, L Zhang, R Hickman, JM Hove, Appl. Phys. Lett. 75, 2569-2571 (1999).

\section{FIGURES}
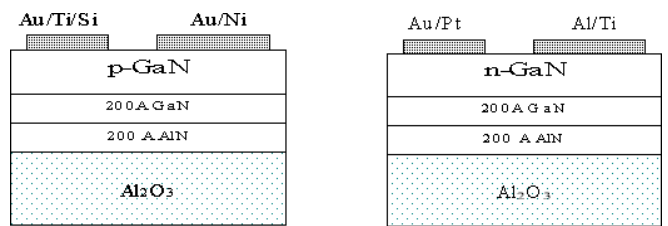

Figure 1. Schematic of $\mathrm{n}$ and $\mathrm{p}-\mathrm{GaN}$ Schottky structures.

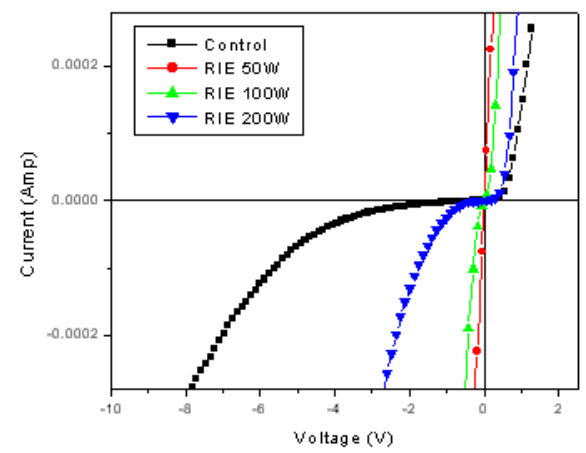

Figure 2a. I-V characteristics from n-GaN exposed to RIE for $30 \mathrm{sec}$ at different RF powers. I-V characteristics of control samples are also shown.

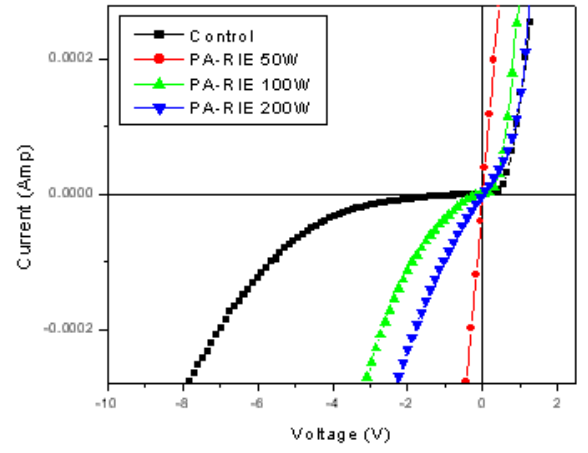

Figure $2 b$. I-V characteristics from n-GaN exposed to RIE for $30 \mathrm{sec}$ at different PA-RIE for the same conditions. I-V characteristics of control samples are also shown.

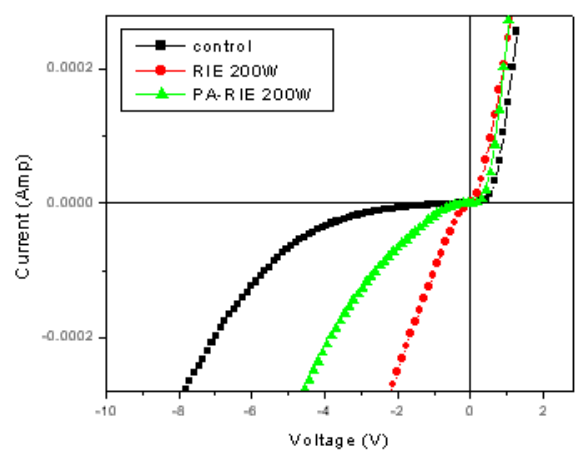

Figure 3. I-V characteristics of n-type GaN-based Schottky diodes fabricated using RIE and PA-RIE for 2 min at 200W RF power (-300 self dc-bias), $30 \mathrm{mTorr}$ and $10 \mathrm{sccm} \mathrm{Cl} \mathrm{Cl}_{2} / 10 \mathrm{sccm}$ $\mathrm{BCl}_{3} / 10 \mathrm{sccm} \mathrm{N}$. I-V characteristic of control sample is also shown. 


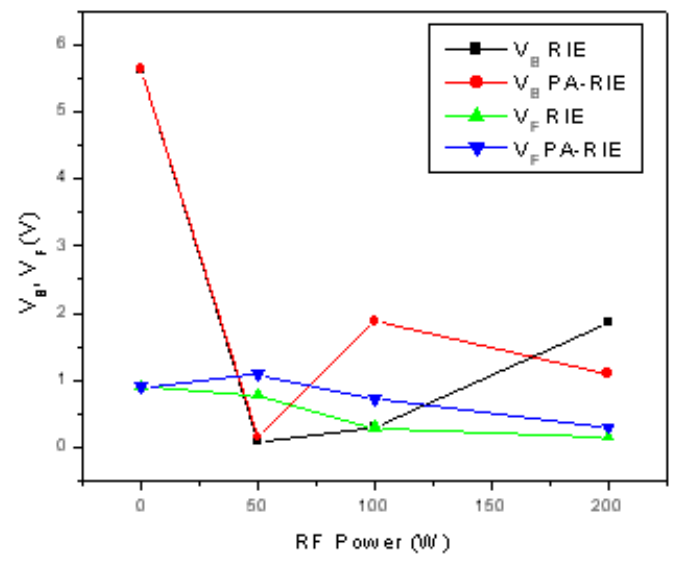

Figure 4. Variation of reverse breakdown voltage $\left(\mathrm{V}_{\mathrm{B}}\right)$ and forward turn-on voltage $\left(\mathrm{V}_{\mathrm{F}}\right)$ as a function of RF power for $n$ type GaN-based Schottky diodes processed by RIE and PA-RIE for $30 \mathrm{~s}$ using $10 \mathrm{sccm} \mathrm{Cl}_{2} / 10 \mathrm{sccm} \mathrm{BCl}_{3} / 10 \mathrm{sccm} \mathrm{N}$ at 30 mTorr. $V_{B}$ and $V_{F}$ of control sample are also shown. Lines are a guide to the eye only.

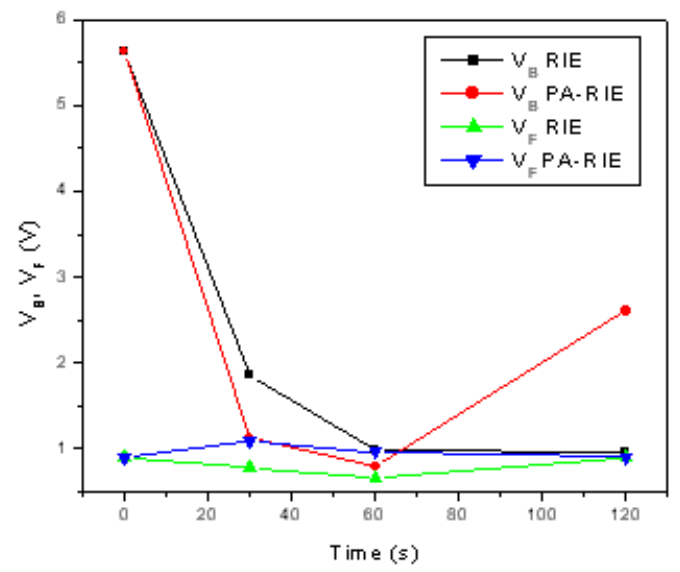

Figure 5. Variation of reverse breakdown voltage $\left(\mathrm{V}_{\mathrm{B}}\right)$ and forward turn-on voltage $\left(\mathrm{V}_{\mathrm{F}}\right)$ as a function of etch time for $n$ type GaN-based Schottky diodes processed by RIE and PA-RIE at $200 \mathrm{~W}$ RF power using $10 \mathrm{sccm} \mathrm{Cl}_{2} / 10 \mathrm{sccm} \mathrm{BCl}_{3} / 10 \mathrm{sccm}$ $\mathrm{N}_{2}$ at 30 mTorr $\left(\mathrm{V}_{\mathrm{B}}\right.$ and $\mathrm{V}_{\mathrm{F}}$ of control sample are also shown).

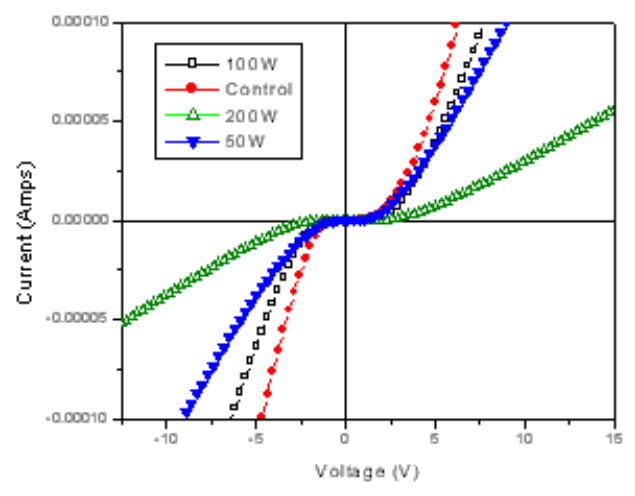

Figure 6. I-V Characteristics from $\mathrm{p}-\mathrm{GaN}$ structures exposed to $\mathrm{Cl}_{2} / \mathrm{N}_{2}$ RIE at $30 \mathrm{mTorr}$ for $3 \mathrm{~min}$ as a function of RF power. 